\title{
Quality Of Selected Anti-retroviral Medicines: Tanzania Mainland Market As A Case Study
}

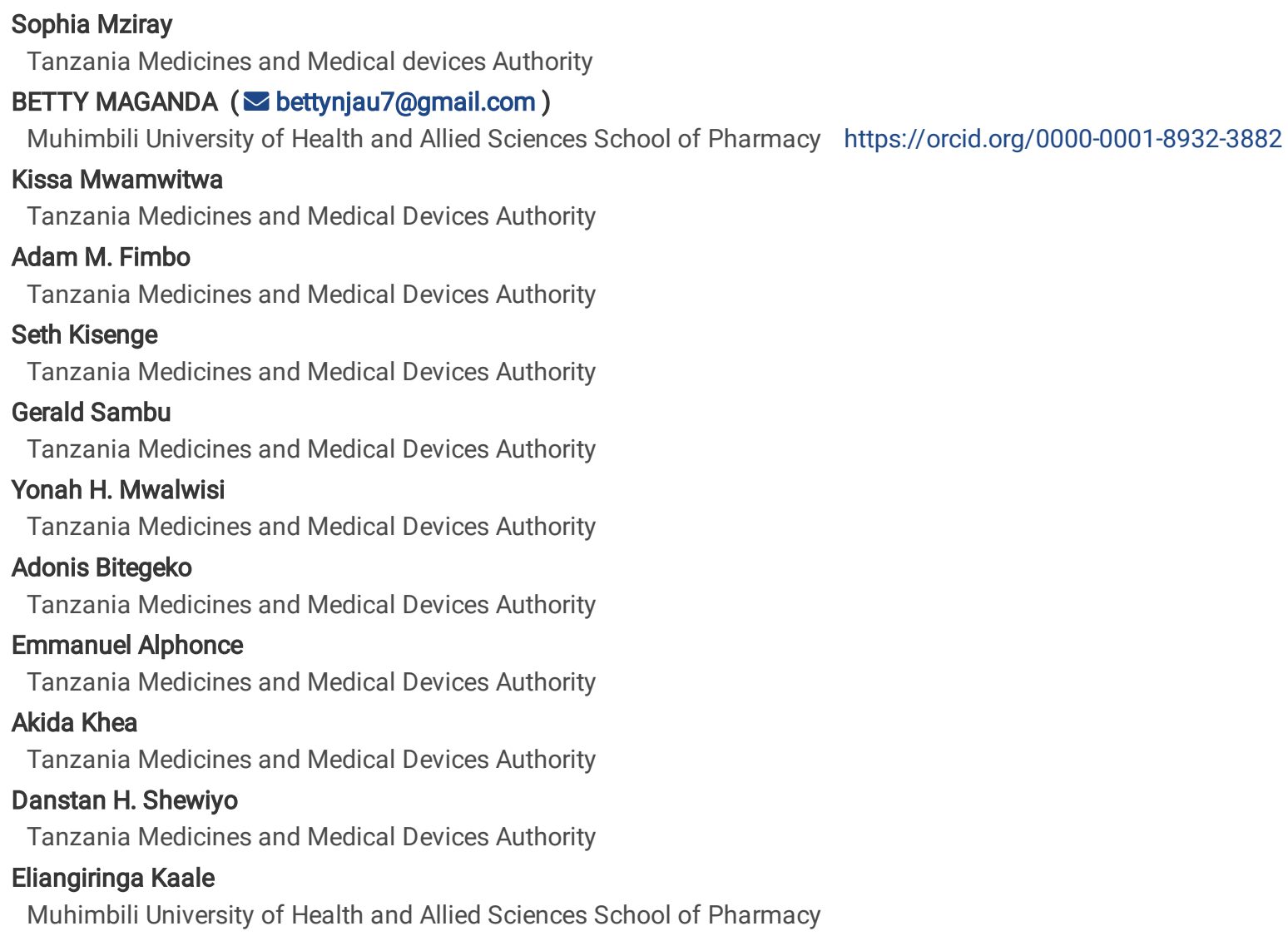

\section{Research article}

Keywords: Medicines quality, Substandard, Falsified, Post market surveillance, antiretroviral medicines, Patient information leaflet Posted Date: March 21st, 2020

DOI: https://doi.org/10.21203/rs.3.rs-18179/v1

License: (c) (i) This work is licensed under a Creative Commons Attribution 4.0 International License. Read Full License 


\section{Abstract}

Background Human Immunodeficiency Virus and Acquired Immune Deficiency Syndrome (HIV/AIDS) is still health problem worldwide, particularly in sub-Saharan African countries, such as Tanzania. Anti-retroviral medicines (ARVs) have significantly reduced morbidity, mortality and improved the quality of life of people living with HIV infection. Thus, good quality ARVs is amongst the key factors which guarantee successful therapeutic outcomes and prevention of development of drug resistant strains. This study was aimed at monitoring the quality of ARVs circulating on the Tanzania Mainland market.

Methodology This was a prospective cross sectional study. ARVs samples were collected in 20 regions of Tanzania Mainland, between 2012 and 2018. All samples were subjected to screening testing using the Global Pharma Health Fund ® Mini-Lab kits. Sampled ARV's that failed screening test or yielded doubtful results and ten percent (10\%) of all that complied with the screening test requirements were subjected to confirmatory testing using full pharmacopoeia monographs at the Tanzania Medicines and Medical Devices Authority (TMDA), quality control laboratory prequalified by the World Health Organisation. Samples collected from the medicine distribution outlets were also subjected to product information review.

Results A total of 2,630 samples were collected, of which $83.7 \%$ (2200/2630) were from port of entry (POEs). Out of the 430 samples which were subjected to product information review, $25.6 \%$ (110/430) failed to comply with the packaging and labelling requirements. All sampled ARV's that were screened and conformed to the specifications, except of the fixed dose combination (FDC) lopinavir/ritonavir $0.27 \%$ (7/2630) and lamivudine/zidovudine/nevirapine $0.27 \%$ (7/2630) that failed the disintegration test. In confirmatory testing using full pharmacopoeia monographs, all samples conformed to the requirements, except of FDC stavudine/lamivudine/nevirapine which failed disintegration $2 \%$ $(2 / 100)$ and assay tests $1 \%(1 / 100)$ respectively. Samples failing the assay test had low content of stavudine (86.6\%) versus specification limits $(90 \%-110 \%)$.

Conclusion The quality of majority of ARVs circulating on the Tanzania Mainland market was good. The existence of number of ARVs circulating on Tanzania Mainland market which do not comply with product information requirements was significant. These results call for continuous monitoring of quality of medicines circulating on the Tanzania market.

\section{Background}

Human immunodeficiency virus (HIV) infection and acquired immunodeficiency syndrome (AIDS) are still major global health problem particularly in sub-Saharan Africa [1, 2]. Besides being medical problem, HIV/AIDS affects other aspects of life such as economy social and demography [1]. The joint United Nations programme on HIV/AIDS (UNAIDS) estimated that by the end of 2017, 36.9 million [31.1 million-43.9 million] people worldwide were living with HIV/ AIDS [1, 2, 3]. Sub-Saharan Africa is the world's most affected region with an estimation of 25.6 million people living with HIV/AIDS [2]. Globally, HIV/AIDS has remained one of the leading causes of morbidity in the last three decade [4, 5]. The prevalence of HIV/AIDS in Tanzania for people aged between 15-64 years is 5\% [1, 4]. Recent data shows that, 1.4 million people are living with HIV/AIDS in Tanzania [1, 4].

According to UNAIDS and World Health Organization (WHO) reports, 21.7 million HIV-infected patients are currently, accessing antiretroviral therapy (ART) worldwide [1-3], with about 1.2 million of them residing in Tanzania [4]. The ART have significantly reduced morbidity and mortality, by preventing relapse and opportunistic infections. Equally, it has vastly improved the quality of life and productivity of HIV-infected patients and has lowered the risk of HIV transmission [6, 7].

ART is mainly responsible for suppressing the viral replication at therapeutic plasma drug level to undetectable levels, allowing satisfactory immune recovery [6-9]. Nonetheless, treatment outcomes in HIV infected patients depends on but not limited to genetic variations in drug metabolizing enzymes, adherence to treatment and quality of medicines, of which may affect therapeutic outcomes.

Scrutiny on the quality of medicines available in Sub Saharan African countries markets has revealed poor quality medicines that are either falsified or substandard [10-23]. This has been documented in a number of countries including Tanzania [10-23]. Poor quality ART poses a major public health threat such as treatment failure, drug toxicity, poor diseases prognosis and emergence of resistant strains, increased comorbidity and healthcare cost to government and individual [12, 22-24].

The UNAIDS report shows that, in Sub Sahara African countries about $6 \%$ of patients receiving first line ART are obliged to switch to second line regimen per year, which are more costly [9]. For this reason, continued monitoring of the quality of ART is of importance as can identify substandard and falsified medicines circulating on the market, thus, preventing them from causing harm to patients.

Therefore, this study was aimed at monitoring the quality of selected ART circulating on the Tanzania Mainland market. The primary objective of this study was to determine the proportion of poor quality ART circulating on the market and secondary was to determine the quality of information on the primary and secondary packaging and availability of patient information leaflet.

Page $2 / 12$ 


\section{Methods}

\section{Study design}

This was a prospective cross sectional study.

\section{Study settings}

This study was conducted in 20 out of 26 regions of Tanzania mainland namely; Morogoro, Tanga, Dar es Salaam, Dodoma, Coast, Simiyu, Njombe, Arusha, Shinyanga, Kagera, Singida, Rukwa, Iringa, Geita, Kilimanjaro, Mara, Mbeya, Mtwara, Mwanza and Ruvuma. The regions were selected based on the pre-defined criteria including, highly populated regions, regions bordering other countries, regions with high prevalence of HIV infection and those reported to have medicine quality problems.

\section{Study duration}

Antiretroviral drugs (ARVs) samples were collected from medicines distribution outlets in 20 regions of Tanzania Mainland, between 2012 and 2015 and port of entry between 2012 and 2018.

\section{ARVs sample size and collection}

Convenience sampling method was used in the collection of selected ARVs. Samples were collected from the port of entry (POEs) and medicines distribution outlets such as Medical Stores Department (MSD), public and private hospitals, dispensaries, health centres, retail and wholesale pharmacies and Accredited Drug Distribution Outlets (ADDO) in two distribution levels. Level I covered POEs and MSD and level II covered public and retail healthcare facilities, wholesale and retail pharmacies and ADDOs. Samples were collected by trained drug inspectors according to the prepared sampling plan and Tanzania Medicines and Medical Devices Authority (TMDA) standard operation procedures. Drug inspectors were able to collect two (2) brands per surveyed medicines at each collection site. All samples were collected in their original containers and information details were filled in the sample collection tool. Each collected sample was coded according to the prescribed coding format. Coded samples with their respective sample collection tool were kept in the labelled sampling envelope and sealed. Storage and handling of samples during collection, transportation and before analysis complied with the manufacturer's instructions and TMDA sample chain of custody procedures.

Samples collected from POEs and medicine distribution outlets were subjected to screening test at TMDA zone laboratories and respective quality assurance (QA) centres. Those from medicine distribution outlets were further subjected to product information review (PIR).

Of the sampled ARVs from medicines distribution outlet, $100 \%$ of them that did not comply with screening testing requirements or yielded doubtful results and $10 \%$ of those that complied with screening test requirements were transported to TMDA-WHO prequalified laboratory at the headquarter office for further analysis.

\section{ARVs selection criteria}

ARVs selected in this surveillance were those frequently prescribed for the management of HIV-infection with main focus on first line regimen as per National Guidelines for Management of HIV/AIDS [25] or those reported to have quality problem. These included the following active pharmaceutical ingredient (API): Efavirenz, nevirapine, lamivudine, zidovudine, abacavir sulphate, tenofovir disoproxil fumarate and fixed dose combination (FDC) containing tenofovir disoproxil fumarate /emtricitabine, lamivudine/zidovudine/nevirapine, lamivudine/stavudine/nevirapine, tenofovir/lamivudine/efavirenz, tenofovir/ emtricitabine/efavirenz and lopinavir/ritonavir.

\section{Site sampling techniques}

A purposive sampling technique was used for the selection of medicine collection sites, from regions and district levels down to the distribution channels.

\section{Quality evaluation}

\section{Product information review}

Prior to further laboratory analysis, samples collected from medicine distribution outlets were subjected to PIR. Each sample was evaluated visually on physical appearance of primary and secondary packaging. Availability and information on package information leaflets (PIL) based on the TMDA labelling requirements and approved product information was also assessed.

Parameters checked during PIR included but not limited to appearance or description of the dosage forms, product's brand and generic name, dosage form and strength, name and address of the manufacturer, batch or lot number, date of manufacturing and expiration, TMDA registration 
number, packaging and pack size, PIL, language and storage instruction. The information was recorded on a designed form.

\section{Tier I laboratory screening testing}

All samples were subjected to screening test using Global Pharma Health Fund® (GPHF) Mini-Lab kits manual. The performed tests were simple visual inspection, disintegration for solid dosage forms and identification test using Thin Layer Chromatography (TLC) method. Oral solutions were also tested for $\mathrm{pH}$ appropriateness and microbial limit as described in the United States Pharmacopoeia (USP, NF 32).3

\section{Visual inspection}

All sampled solid dosage forms were checked for uniformity of shape, physical damage, altered surface, odor, color uniformity and dirty marks. Liquid dosage forms were examined for clarity, odor and colour uniformity.

\section{Simple disintegration test}

A simple disintegration test was performed to assess the possibility of instant-release of oral solid dosage forms. This was done by using a $100 \mathrm{~mL}$ wide neck glass bottle filled with water heated to $37 \mathrm{oC}$. Tablets were shaken or stirred occasionally. Disintegration was required to occur within 30 minutes. The sample was considered failed if did not disintegrate within 30 minutes in three (3) consecutive independent tests.

\section{Product identification by thin layer chromatograph}

TLC method was used for product identification and qualitative determination of active ingredients, related substances and impurities present in the dosage forms. This method employed the principle of comparing spots test sample and reference solutions according to GPHF Minilab. The principal spot obtained with the test sample was required to correspond with the chromatographic runs of the standard solution in terms of colour, shape, size, intensity and retardation factor (Rf) value. The test sample was considered failed if the Rf value of the test sample was different by more than $10 \%$ from that of the standard sample. Also, the sample was considered failed if the intensity of the spot was less than that of a reference containing $80 \%$ of the stated amount of the API and if failure was observed in all three independent experiments.

\section{Tier II laboratory confirmatory testing}

All samples collected from medicines distribution outlets that had failed screening test, $10 \%$ of those which had passed screening test, and those with doubtful results were subjected to confirmatory testing. Confirmatory testing was carried out at the TMDA-WHO prequalified laboratory as per USP pharmacopoeia monograph requirements [26] and/or in house specifications where no official pharmacopoeia monograph existed. Typical parameters tested were physical appearance, identification, disintegration, dissolution, assay, related substances, weight uniformity and microbial limit.

\section{Data management and analysis}

The collected data were checked for any inconsistencies. The data was double-entered into a Microsoft Access database, verified and exported to SPSS (version 20) software for analysis. Descriptive statistics was used and results for PIR, screening and conformity were expressed in percentage form.

\section{Results}

\section{Antiretroviral samples collected}

A total of 2,630 ARVs samples were collected between 2012 and 2018 from POEs and medicine distribution outlets in Tanzania Mainland. Large quantity of samples $83.7 \%(2,200 / 2,630)$ were collected from POEs as depicted in Figure 1.

ARVs samples from POEs were collected in large quantity in the years 2014, 2015, 2016 and 2017, 77\% (1,695/2,200) compared to other years, as shown in Figure 2.

Regarding, the number of samples from different medicine distribution outlets, hospitals ranked highest amounting to $61 \%$ (262/430) as portrayed in Figure 3. Regional wise, 30\% (129/430) were sampled from Dar es Salaam, Mwanza and Iringa and least quantity 1.4\% (6/430) was collected from Kagera.

Thirteen generic brands ( $6 \mathrm{API}$ and 7 fixed dose combinations) contributed to the 430 samples collected from the medicine distribution outlets. The FDC of lamivudine/zidovune/nevirapine tablets was collected in large quantity $17 \%$ (73/430) followed by lamivudine/zidovudine tablets combination $16.3 \%(70 / 430)$ as compared to other ARVs. The proportions are summarized in Table 1 below:

\section{Quality evaluation}




\section{Product information review}

In this study only samples collected from medicine distribution outlets were subjected to PIR and the results are summarized in Figure 4 . Of the examined samples, $25.6 \%$ (110/430) did not comply with the packaging information requirements. The highest failure rate was found in the samples collected in the year 2012, 53\% (44/83). Interesting observation was found in the year 2013, whereas, all samples passed the PIR assessment. Inappropriate or lack of storage condition, lack of the name and address of the manufacturer, discrepancy of address of the manufacturer on the primary and secondary packaging and lack of package insert were the common deficiencies. On the other hand, all sampled medicines were found to be registered in Tanzania, but some of labels did not indicate the Tanzania registration numbers. The trend is comparable to recent studies conducted on antimalarial and antihypertensive drugs $(10,11)$.

\section{Laboratory tier I screening testing}

All ARVs, sampled from POEs conformed to tier I screening test requirements. All sampled ARVs from medicine distribution outlets conformed to visual appearance and identification test requirements. However, 3.3\% (14/430) of the samples from the medicines distribution outlets, failed disintegration test; these were FDC of lopinavir/ritonavir tablets (7/430) and lamivudine/zidovudine/nevirapine tablets (7/430).

\section{Laboratory tier II confirmatory testing}

Of the 430 ARVs samples collected from medicine distribution outlets and subjected to tier I screening testing, 100 of them were subjected to tier II confirmatory test. These samples included all those failed screening tests, $10 \%$ of those which passed screening test and those whose results were doubtful as depicted in Table 2.

Majority of samples $94 \%$ (94/100) complied with confirmatory test requirements. However, $3 \%$ (3/100) failed confirmatory test and $3 \%$ (3/100) samples were beyond expiry dates at the time of analysis, hence, were not analyzed. The failed samples were of FDC of stavudine/lamivudine/nevirapine $3 \%(3 / 100)$ which failed disintegration test $2 \%(2 / 100)$ and assay test $1 \%(1 / 100)$, having low content of stavudine (86.6\%) of specified amount (limit 90\% -110\%). This necessitated more samples from the same manufacturer to be subjected to confirmatory test as described in the USP. The additional samples also failed assay test with results ranging between 86.4 and $87.1 \%$. This confirmed the non conformity of stavudine against the specified requirements. These results are summarized in Figure 5.

\section{Discussion}

Quality medicines are essential to promote public health and disease management. However, various studies conducted worldwide have reported availability of poor quality medicines in the market including ARVs, mostly in developing countries such as Tanzania [10-23].

This survey assessed the quality of 2,630 ARVs samples, collected from medicine distribution outlets and POE. A total of twenty (20) regions representing more than $76 \%$ of the regions of Tanzania Mainland were selected for the purpose. The selection of the regions to be surveyed included some regions bordering other countries; frequency of inspection by the regulatory bodies; areas previously reported to have medicines quality problems and HIV prevalence patterns as indicated in HIV/AIDS and malaria indicator survey of 2011/12 and HIV impact survey of 2016/17 [24, 25]. In this survey, 30\% (129/430) of samples from medicine distribution outlets were collected in three regions namely Mwanza, Dar es Salaam and Iringa matching with high prevalence of HIV infection [4, 27].

It is clear from our results that, majority $83.7 \%$ (2200/2630) of the samples were collected from POE showing the dependence of the country on imported medicines. This observation is in agreement with TFDA data of 2017 [28] and well supported with results from this study that among of the collected samples none were produced locally.

A significant increase of ARVs sampled from the POE in the year 2014, 2015, 2016 and 2017 compared to 2012/13 can be associated with the scaling up of ARVs access in low and middle income countries through the WHO and Global Fund initiatives [29]. Improved access to ARVs has accounted to the levelling off of new HIV infections cases, deaths and life longevity amongst HIV-infected patients [1-3, 9].

Despite the inclusion of ADDO and pharmacies among the medicine distribution outlets, no sample was obtained from these sources. This is an interesting finding on compliance to requirements on availability of ARVs in specialized HIV-clinics, hospitals, health centres and dispensaries [29].

Among the collected FDC samples, oral solid dosage forms were the majority (99.8\%). FDCs are desirable and recommended by the WHO, as it simplify treatment, ideally resulting in improved medication concordance, clinical outcomes and quality of life of patients [7]. The FDC of lamivudine/zidovudine/nevirapine ranked highest (17\%) amongst other ARVs. This was a default regimen during the survey period which is reflected by its wide collection [30]. 
Samples collected from the POEs were not subjected to PIR. This is because, they were being collected on daily basis within the framework of medicines quality assurance system in which the first step is laboratory screening. On the other hand, 25.6\% (110/430) of the sampled ARVs from medicines distribution outlets, subjected to PIR failed to comply with requirements. The failure rate was highest in 2012 , but decreased significantly in subsequent years, as shown in Fig. 3. Adherence to regulatory requirements of individual countries as well as harmonization efforts on technical requirements across the regions could explain the continuing improvement. Compared with PIR results for other medicine categories in the implementation of PMS programs, these results are far much better [10, 11, 31].

All pharmaceutical products must be stored under conditions provided by manufacturers to ensure that their potency and qualities are not compromised during the distribution process and storage. In this study it was observed that some of the manufacturers did not indicate proper storage conditions while some provided conditions that are not achievable in tropical set up, such as "store below $25^{\circ} \mathrm{C}$ ". In country like Tanzania which fall under ICH zone IVb climatic condition it is not easy to achieve/maintain the aforementioned storage condition [32]. Improper storage of pharmaceutical products could negatively impact on their potency, quality, efficacy, safety and subsequently compromising the quality of life of the end users [33, 34].

Information on package inserts is necessary since it is associated with drug safety, adherence to treatment, rational drug use and reporting adverse drug reactions [35]. Our findings revealed absence of package inserts from majority of the collected samples.

This situation poses potential risk to patients on poisoning, adverse drug reactions, development of window for drug resistant strains, as well as treatment failure due to lack of important information [35].

All ARVs sampled in this study were registered in Tanzania but the registration number which is a prerequisite was not indicated on the labels to some packages. The Tanzania registration number enables easy identification of pharmaceutical products authorized by TMDA to be in the market and prevents availability of falsified products on the market. These results are consistent with PIR results from previous survey study conducted in Tanzania for other categories of medicines [10, 11].

The FDC of lamivudine/zidovudine/nevirapine and lopinavir/ritonavir tablets were the only ARVs that failed in the Tier I screening test accounting to $0.53 \%$ (14/ 2630) of the failure rate. Passing Tier II confirmatory test for these samples provided evidence that were of good quality. The situation which stresses on the need for confirmatory tests before conclusion can be made on quality of medicines. The cause of failure in the screening tests for only products sampled from medicine distribution outlets may be due to poor storage conditions. Improper storage of ARVs is known to decrease the potency and quality of pharmaceutical product $[33,34,36]$.

Poor quality ARVs is associated with therapeutic or prophylactic failure and the emergence of resistant strains of HIV [37, 38]. In confirmatory testing by full monograph, all samples tested met the specifications except two (2) samples of FDC of stavudine/lamivudine/nevirapine which failed disintegration test and one (1) sample of the same which failed assay test. A sample which failed the assay specifications had stavudine content below the acceptance criteria. This was confirmed by the consistency of results obtained from repeated analysis done in triplicate. As a point of information, the stavudine concentration is no longer an issue of discussion since ARVs regimen containing stavudine has been phased out in the country due to its severe toxicity [39-41].

Disintegration of tablets within 30 minutes predicts dissolution within the required time in vivo [42], and is a precondition to drug absorption process. Poor absorption of the medicine is associated with poor therapeutic outcomes and development of resistant strains. In spite of the fact that the failure rate was only $2 \%$, it cannot be ignored because presence of substandard drugs in the market at any percentage poses threats to society. These threats are as follows but not limited to drug toxicity, poor treatment outcomes and disease prognosis, drug resistance and death but also in terms of economic implications and effects on global pandemics [43].

Results for assay and disintegration tests in this study are consistent with reports from various studies worldwide, which have shown that failure rates for ARVs, is very low [14, 22, 44]. This can be explained by the fact that most of ARVs in the market are procured by government agencies with donor funding. The funding agencies impose stringent procurement condition such as requirements for the WHO pre-qualification for all suppliers and so, low failure rates [43]. Of importance is that for all sampled medicines that were deemed to have deficiencies during PIR, the TMDA directed manufacturers to bring requests for amendments for all deficiencies observed. Also, the manufacturers and local agents were informed to recall all batches that failed the confirmatory test from the market. The manufacturers were further directed to halt importation of such products and to conduct thorough investigations to identify possible causes of the failure.

\section{Conclusion}

The quality of the majority of ARVs surveyed was good. This results indicates that the efforts made by the WHO and other organizations on prequalification and purchase policies on ARVs have a positive effect. The study also evidenced the existence of a significant number of ARVs circulating on the Tanzania Mainland market which do not meet packaging information requirements. Product information is crucial for proper handling and use of medicinal products. Thus, lack of and/or inadequate information on the package of the products could have negative 
impact on the lives of the intended users. These results call for continuous monitoring of quality of medicines circulating on the Tanzanian market with much emphasis on the adequate information on packages leaflets.

\section{Abbreviations}

ADDO: Accredited Drug Dispensing Outlet; MSD: Medical Stores Department;

TMDA : Tanzania Medicines and Medical Devices Authority; TLC: Thin Layer Chromatography; ARVs: Antiretroviral drugs; WHO: World Health Organization; POEs: Port of entries.

\section{Declarations}

\section{Ethics and consent to participate}

Not applicable

\section{Consent for publication}

Not applicable

\section{Availability of data and materials}

All relevant data generated and analyzed during this study are available from the Director General of TMDA on reasonable request.

\section{Competing interests}

The Authors declared that no competing interest.

\section{Funding}

We declare that the study was funded by Global Funds (GF) and TMDA government funds.

\section{Authors' contributions}

SM and KM contributed in study conception and design, data collection, analysis and manuscript writing, SK contributed in data collection, analysis and manuscript writing. BAM contributed in data analysis and manuscript writing and proof reading. GS, YHM and DHS contributed in laboratory and data analysis and manuscript writing, whereas $A B$ and EA contributed in data collection and manuscript writing. Lastly, AF, AK and EK contributed in writing and proof reading the manuscript. All authors participated in reading and approving the final manuscript.

\section{Acknowledgements}

We wish to express our heartfelt appreciation to all parties involved in this activity particularly; Department of Pharmacovigilance and Clinical Trial at TMDA which coordinates post market surveillance activities. Drug inspectors who took part in sample collection, evaluator's who reviewed the product information and analysts who carried out laboratory testing are highly thanked.

\section{References}

1. UNAIDS data. 2017. http://www.unaids.org/sites/default/files/media_asset/20170720_Data_book_2017_en.pdf. Accessed 25 June 2019.

2. World Health Organization. HIV/AIDS key facts. 2019. http://www.who.int/news-room/fact-sheets/detail/hiv-aids. Accessed 25 June 2019.

3. Global HIV \& AIDS statistics-fact sheet. 2018. www.unaids.org/en/resources/fact-sheet. Accessed 18 January 2019.

4. Ministry of Health Tanzania. Tanzania HIV impact survey (THIS) 2016-2017. 2017. http://phia.icap.columbia.edu/wpcontent/uploads/2017/11/Tanzania_SummarySheet_A4.English.v19.pdf. Accessed 2 June 2019.

5. The United Republic of Tanzania: Global AIDS response country progress report. 2014. http://www.unaids.org/sites/default/files/country/documents/TZA_narrative_report_2014. Accessed 30 August 2019

6. United State of America Food and drug administration (FDA). Antiretroviral drugs used in the treatment of HIV infection. http://www.fda.gov/ForPatients/IIIness/HIVAIDS/Treatment/ucm118915.htm. Accessed 4 September 2019

7. Consolidated guidelines on the use of antiretroviral drugs for treating and preventing HIV infection. Recommendations for a public health approach. Second edition. 2016. 
https://apps.who.int/iris/bitstream/handle/10665/208825/9789241549684_eng.pdf;jsessionid=B3C358E06E50A996385D3778BB625263? sequence=1. Accessed 20 June 2019.

8. Laurent C, Ngom Gueye NF, Ndour CT, Gueye PM, Diouf M, Diakhaté N, et al. Long-term benefits of highly active antiretroviral therapy in Senegalese HIV-1-infected adults. J Acquir Immune Defic Syndr. 2005;38(1):14-7.

9. Universal Access to HIV Prevention, Treatment, Care and Support. From Countries to Regions to the High Level Meeting on AIDS and Beyond 2011 Road Map. https://www.unaids.org/sites/default/files/sub_landing/files/2011_UA_roadmap_en.pdf. Accessed 20 June 2019.

10. Mziray S, Mwamwitwa K, Kisoma S, Augustine S, Fimbo A. Post Marketing Surveillance of Anti-malarial Medicines in Tanzania. Pharm Regul Aff. 2017; 6(191):2.

11. Mwamwitwa K, Fimbo A, Mziray S, Nandonde M, Hipolite D, Kijo AS, Njau B and Kaale E. Post Marketing Surveillance of Antihypertensive Medicines in Tanzania. Pharmaceut Drug Regul Affair J. 2018; 1: 1-10.

12. Counterfeit Medicines: an update on estimate. 2006.

http://www.who.int/medicines/services/counterfeit/impact/TheNewEstimatesCounterfeit.pdf Accessed 22 June 2019.

13. Survey of the quality of selected antimalarial medicines circulating in six countries of Sub-Sahara Africa. 2011. http://apps.who.int/medicinedocs/documents/s17835en/s17835en.pdf. Accessed 28 August 2019.

14. Primo-Carpenter J, McGinnis M. Matrix of Drug Quality Reports in USAID-assisted Countries by the U.S. Pharmacopeia Drug Quality and Information Program. 2006. https://pdf.usaid.gov/pdf_docs/Pnadh099.pdf. Accessed 20 June 2019.

15. Hajjou M, Krech L, Lane-Barlow C, Roth L, Pribluda VS, Phanouvong S et al. Monitoring of Quality of medicines - results from Africa, Asia and South America. Am J Trop Med Hyg. 2015; 92 (6): 68-74.

16. Almuzaini T, Choonara I, Sammons H. Substandard and counterfeit medicines: a systematic review of the literature. BMJ Open. 2013; 3 : e002923.

17. Chikowe I, Osei-Safo D, Harrison J, Konadu D, Ivan Addae-Mensah I. Post-marketing surveillance of anti-malarial medicines used in Malawi. Malaria Journal. 2015; 14:127.

18. Osei-Safo D, Agbonon A, Konadu DY, Harrison JJ, Edoh M, Gordon A et al. Evaluation of the Quality of Artemisinin-Based Anti-malarial Medicines Distributed in Ghana and Togo. Malar Res Treat. 2014; 2014: 806416.

19. Lalani M, Kaur H, Mohammed N, Mailk N, Wyk AV, Jan S et al. Substandard Anti-malarials Available in Afghanistan: A Case for Assessing the Quality of Drugs in Resource Poor Settings. Am J Trop Med Hyg. 2015; 92 (6): 51-8.

20. Jande M, Ngassapa O, Kibwage I. Quality of sulfadoxine/ pyrimethamine tablets marketed in Dar es Salaam, Tanzania. East Cent Afr J Pharm Sci. 2000; 3: 20-4.

21. Minzi OM, Moshi MJ, Hipolite D, Massele AY, Tomson G, Ericsson O et al. Evaluation of the quality of amodiaquine and sulphadoxine/pyrimethamine tablets sold by private wholesale pharmacies in Dar Es Salaam, Tanzania. J Clin Pharm Ther. 2003; 28: 11722.

22. Survey of the quality of antiretroviral medicines circulating in selected African countries. 2007. http://apps.who.int/medicinedocs/documents/s14230e/s14230e.pdf. Accessed 8 September 2019.

23. Mcginnis MA. Media Reports on Medicine Quality: Focusing on USAID-assisted Countries. By the Promoting the Quality of Medicines program. 2013. http://www.globalforumljd.org/sites/default/files/docs/library/USAID\%20PQM\%202013\%20\%20Media\%20Reports\%20on\%20Medicines\%20Quality.pdf. Accessed 21June 2019.

24. Renschler PJ, Walters KM, Newton PN, Laxminarayan R. Estimated Under-Five Deaths Associated with Poor-Quality Anti-malarials in SubSaharan Africa. Am J Trop Med Hyg. 2015; 92 (6): 119-126.

25. National guidelines for the management of HIV and AIDS. National AIDS Control Programme. 2012. https://aidsfree.usaid.gov/sites/default/files/hts_policy_tanzania.pdf. Accessed 6 July 2018.

26. United State Pharmacopeia (USP) NF 32 Rockville, MD, USA 2012-2014

27. Ministry of Health of Tanzania. HIV/AIDS and malaria indicator survey of 2011/12. 2012. https://dhsprogram.com/pubs/pdf/AIS11/AIS11.pdf. Accessed 20 June 2019

28. Tanzania Food and Drugs Authority Registration Data Base, 2017.

29. The importance of HIV care and support services. 2016. http://www.unaids.org/en/resources/presscentre/featurestories/2016/december/20161202_HIV-care

30. National guidelines for the management of HIV and AIDS. National AIDS Control Programme. 2009. https://www.who.int/hiv/pub/guidelines/tanzania_art.pdf. Accessed 21 June 2019.

31. Post Marketing Surveillance Report of the Quality of Selected Medicines under phase III- IX of 2011-2013 PMS Program. 2016. Tanzania Food and Drugs Authority, Dar-es-Salaam,Tanzania 
32. Climatic Zones for Stability Studies, http://www.pharmaguideline.com/2010/12/different-climatic-zones-for- stability.html. Assessed on 09 March 2019.

33. Ansari FAA. Study of various storage conditions on the pharmaceutical products and its implementation at retail store. The Pharma Innovation Journal. 2017; 6(9): 475-78.

34. Shafaat K, Hussain A, Kumar B , Ul Hasan R, Prabhat P, Kumar VY. An overview: storage of pharmaceutical products. World journal of pharmacy and pharmaceutical sciences. 2013; 2:2499-515

35. Sillo HB, Masota NE, Kisoma S, Rago L, Mgoyela V, Kaale EA (2018) Conformity of package inserts information to regulatory requirements among selected branded and generic medicinal products circulating on the East African market. PLoS ONE 13(5): e0197490.

36. Shakoor O, Taylor RB, Behrens RH. Assessment of the incidence of substandard drugs in developing countries. Trop Med Int Health. 1997; 2(9):839-45.

37. Arya, C S. Adequate quality of HIV drugs must be ensured. 2002; 324(7331): 235.

38. Taylor RB, Shakoor O, Behrens RH. Drug quality, a contributor to drug resistance? Lancet. 1995; 346(8967):122.

39. Minzi O.M.S., Irunde H, Moshiro C. HIV patients presenting common adverse drug events caused by highly active antiretroviral therapy in Tanzania. Tanzania Journal of Health Research. 2009; 11 (1): 5-10.

40. Franzeck FC, Letang E, Mwaigomole G, Jullu B, Glass TR, Nyogea D, Hatz C, Tanner M, Battegay M. cART prescription trends in a prospective HIV cohort in rural Tanzania from 2007 to 2011. BMC Infect Dis. 2014; 14:90.

41. Palmer M, Chersich M, Moultrie H, Kuhn L, Fairlie L, Meyers T. Frequency of stavudine substitution due to toxicity in children receiving antiretroviral treatment in sub-Saharan Africa. 2013; 27(5):781-85.

42. Marton S, Szentmiklósi P, Rácz I. Correlation between disintegration, dissolution and in vivo absorption rate in the case of compounds with benzyl-isoquinoline structures and its pharmacokinetic aspects. Pol J Pharmacol Pharm. 1984; 36(4):385-95.

43. Ozawa S, Evans DR, Bessias S, Haynie DG, Yemeke TT, Laing SK, Herrington JE. Prevalence and Estimated Economic Burden of Substandard and Falsified Medicines in Low- and Middle-Income Countries: A Systematic Review and Meta-analysis. JAMA Netw Open. 2018; 1(4):e181662.

44. Ministry of Public Health and Sanitation Ministry of Medical Services. Post market survey of antiretroviral medicines in Kenya. 2012. http://apps.who.int/medicinedocs/documents/s21944en/s21944en.pdf. Accessed 20 June 2019.

\section{Tables}

Table 1: Proportions of anti-retroviral medicines sampled from medicine distribution outlets between 2012 and 2015

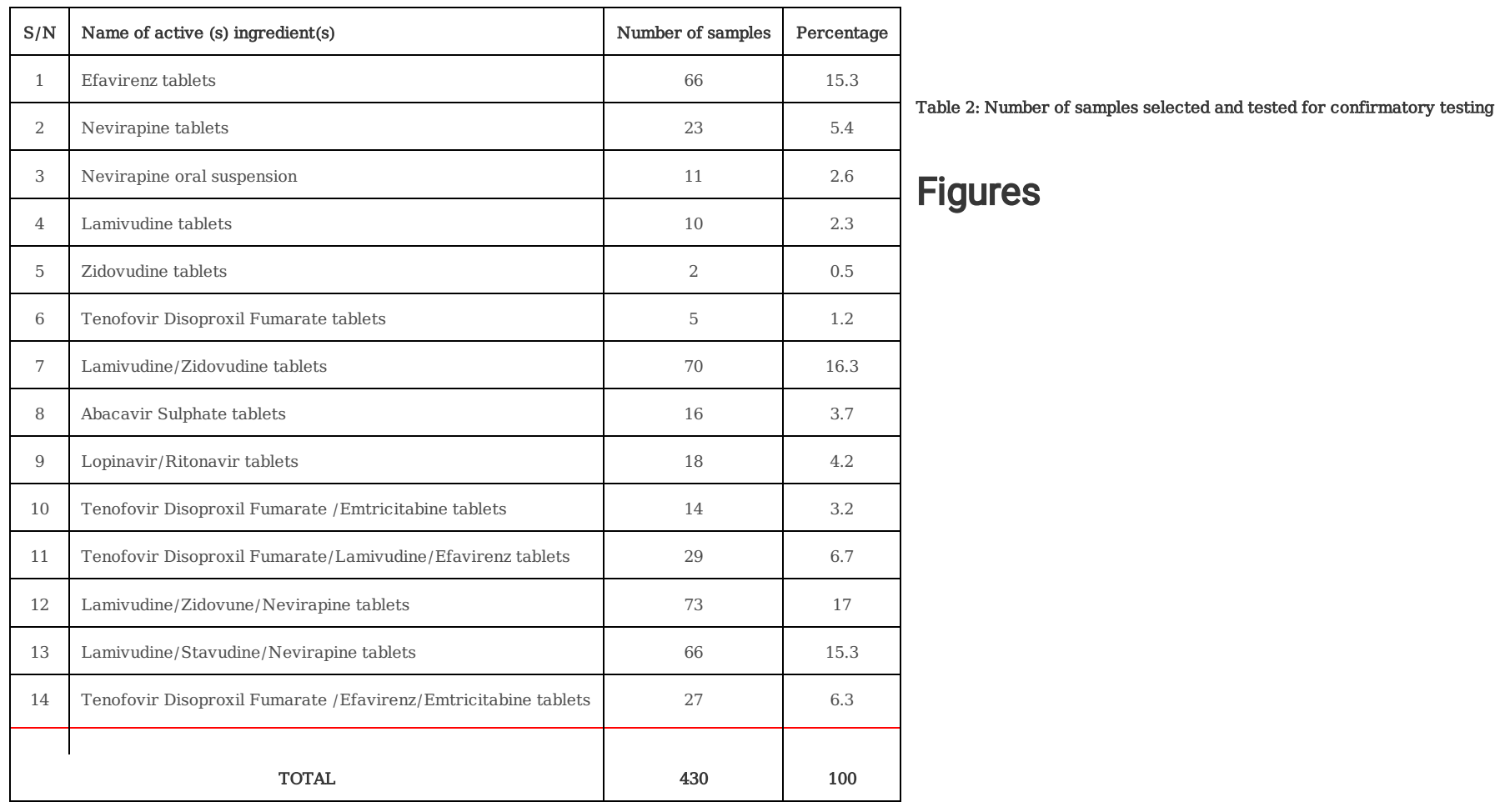




\begin{tabular}{|c|c|c|c|c|c|}
\hline \multirow[t]{2}{*}{ Product name } & \multicolumn{3}{|c|}{ Samples collected and screened } & \multicolumn{2}{|c|}{ Confirmatory test } \\
\hline & $\begin{array}{l}\text { Samples } \\
\text { collected }\end{array}$ & $\begin{array}{l}\text { Samples screened and } \\
\text { passed }\end{array}$ & $\begin{array}{l}\text { Samples } \\
\text { Failed }\end{array}$ & $\begin{array}{l}\text { Samples eligible for } \\
\text { confirmatory }\end{array}$ & $\begin{array}{l}\text { Samples selected and } \\
\text { tested }\end{array}$ \\
\hline Efavirenz & 66 & 66 & 0 & 7 & 14 \\
\hline Nevirapine tablets & 34 & 34 & 0 & 4 & 7 \\
\hline Lamivudine tablets & 10 & 10 & 0 & 1 & 10 \\
\hline Zidovudine tablets & 2 & 2 & 0 & 1 & 2 \\
\hline \multicolumn{6}{|l|}{ Tenofovir Disoproxil Fumarate tablets } \\
\hline & 5 & 5 & 0 & 1 & 5 \\
\hline Lamivudine/Zidovudine tablets & 70 & 70 & 0 & 7 & 7 \\
\hline Abacavir Sulphate tablets & 16 & 16 & 0 & 2 & 4 \\
\hline Lopinavir/Ritonavir tablets & 18 & 12 & 7 & 9 & 9 \\
\hline Tenofovir Disoproxil Fumarate / Emtricitabine tablets & 14 & 14 & 0 & 2 & 4 \\
\hline Tenofovir Disoproxil Fumarate / Lamivudine/Efavirenz & 29 & 29 & 0 & 3 & 6 \\
\hline Lamivudine/Zidovune/Nevirapine tablets & 73 & 73 & 7 & 14 & 14 \\
\hline Lamivudine/Stavudine/Nevirapine tablets & 66 & 59 & 0 & 10 & 10 \\
\hline Tenofovir Disoproxil Fumarate /Efavirenz/Emtricitabine & 27 & 27 & 0 & 3 & 8 \\
\hline Total & 430 & 416 & 14 & 61 & 100 \\
\hline
\end{tabular}

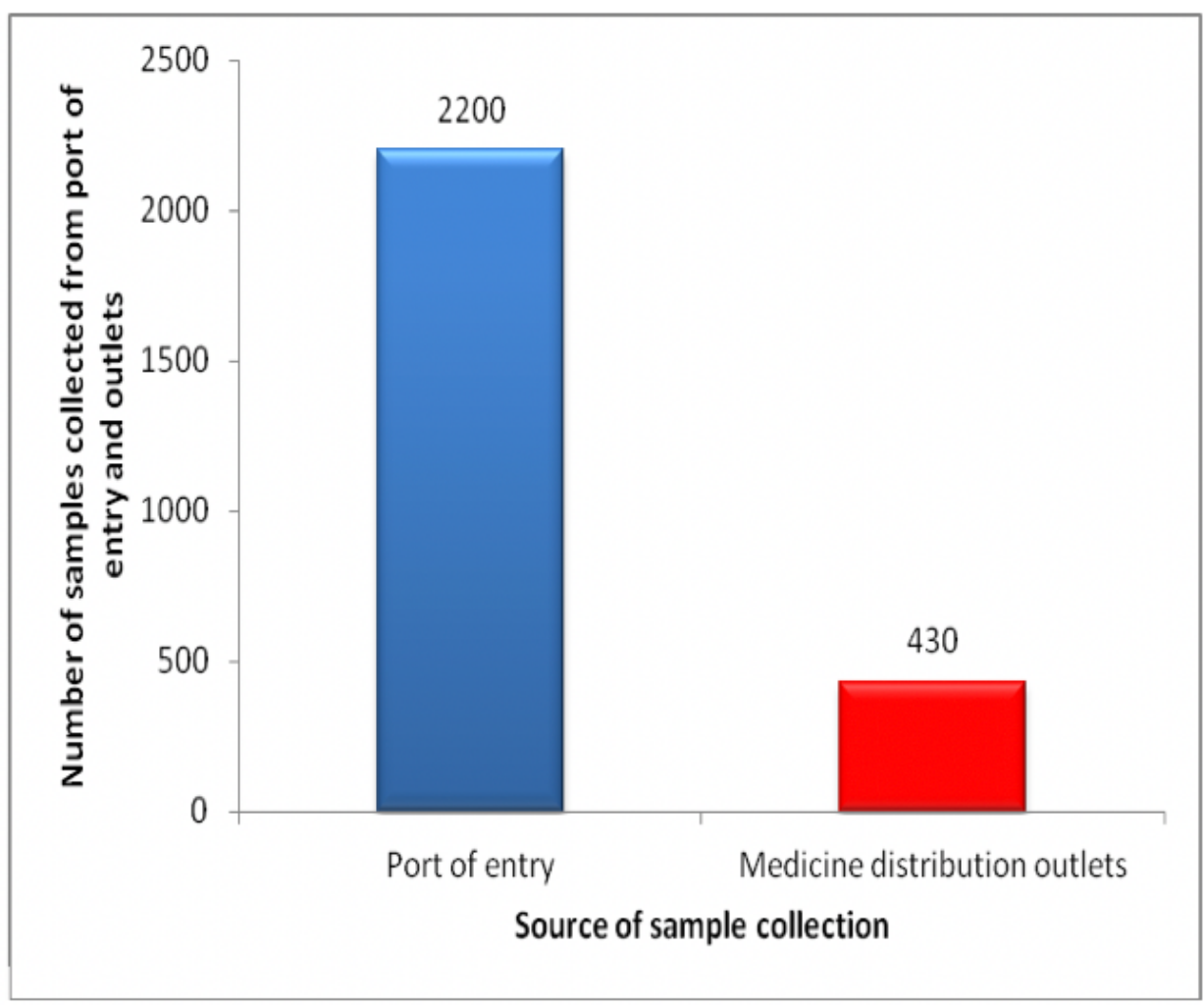

Figure 1

Proportion of antiretroviral drug samples collected from port of entry and medicine distribution outlets

Page $10 / 12$ 


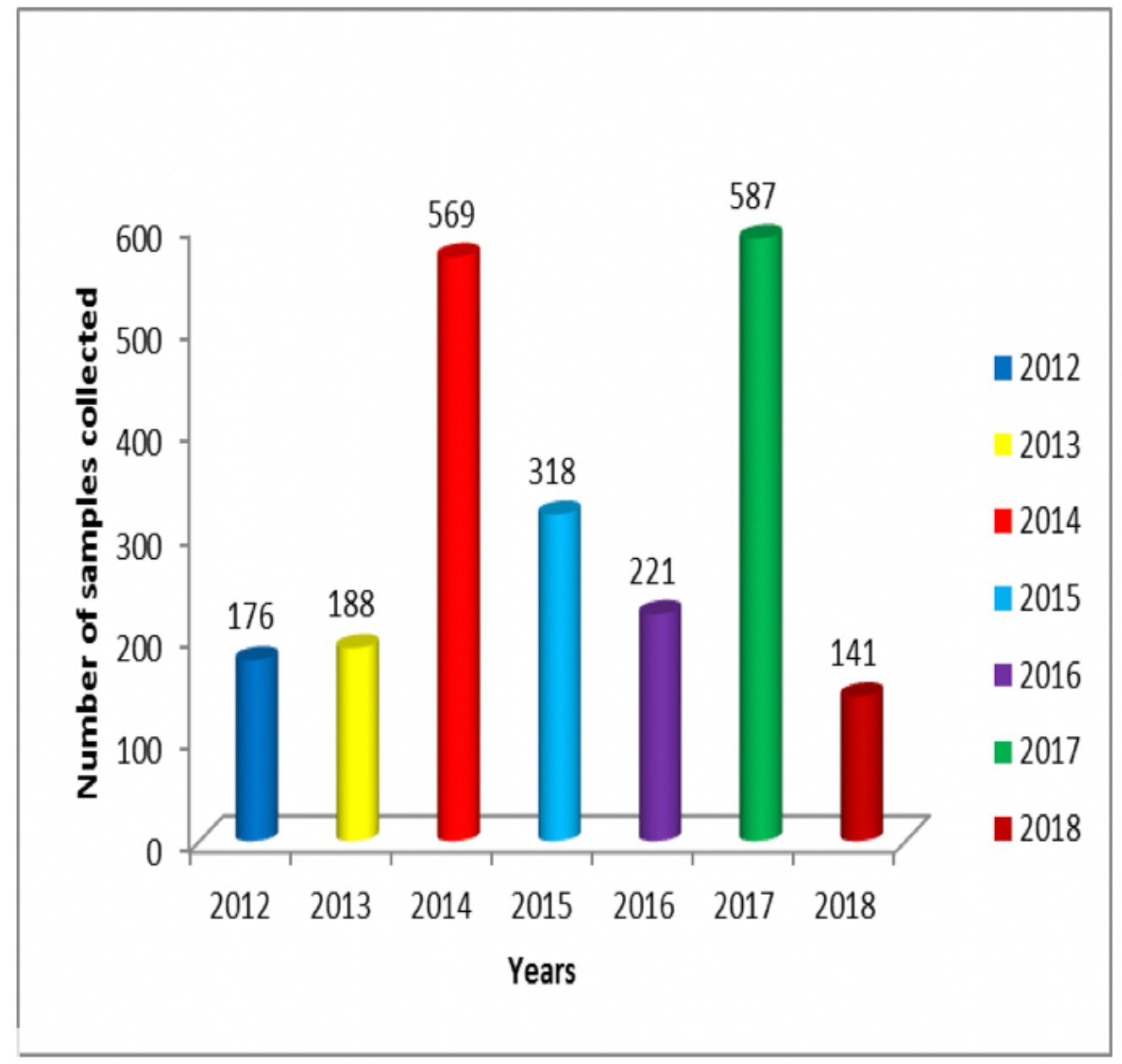

Figure 2

Number of antiretroviral drug samples collected at the port of entry annually (2012 - 2018)

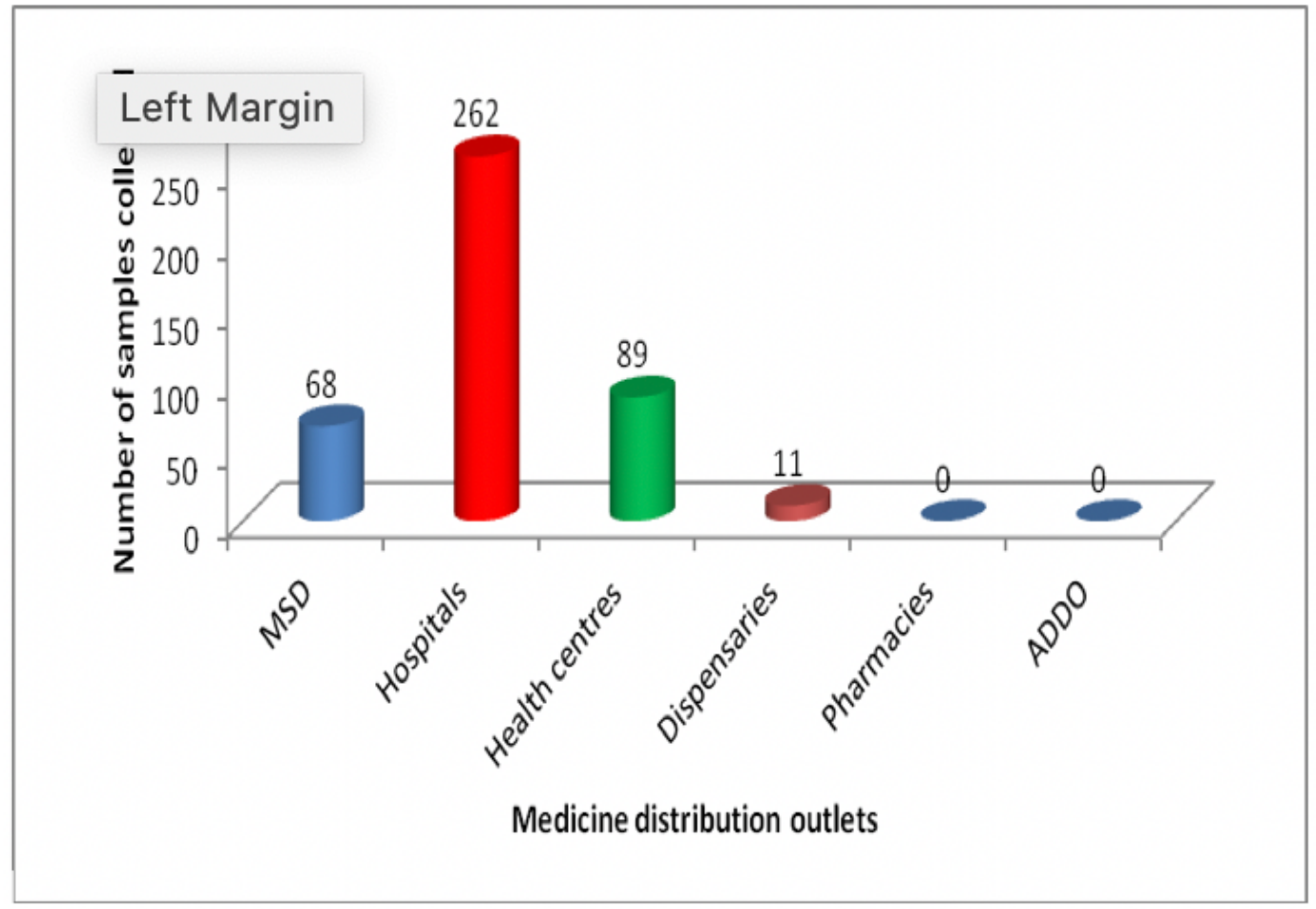

Figure 3 
Samples of antiretroviral drugs collected from different medicine distribution outlets

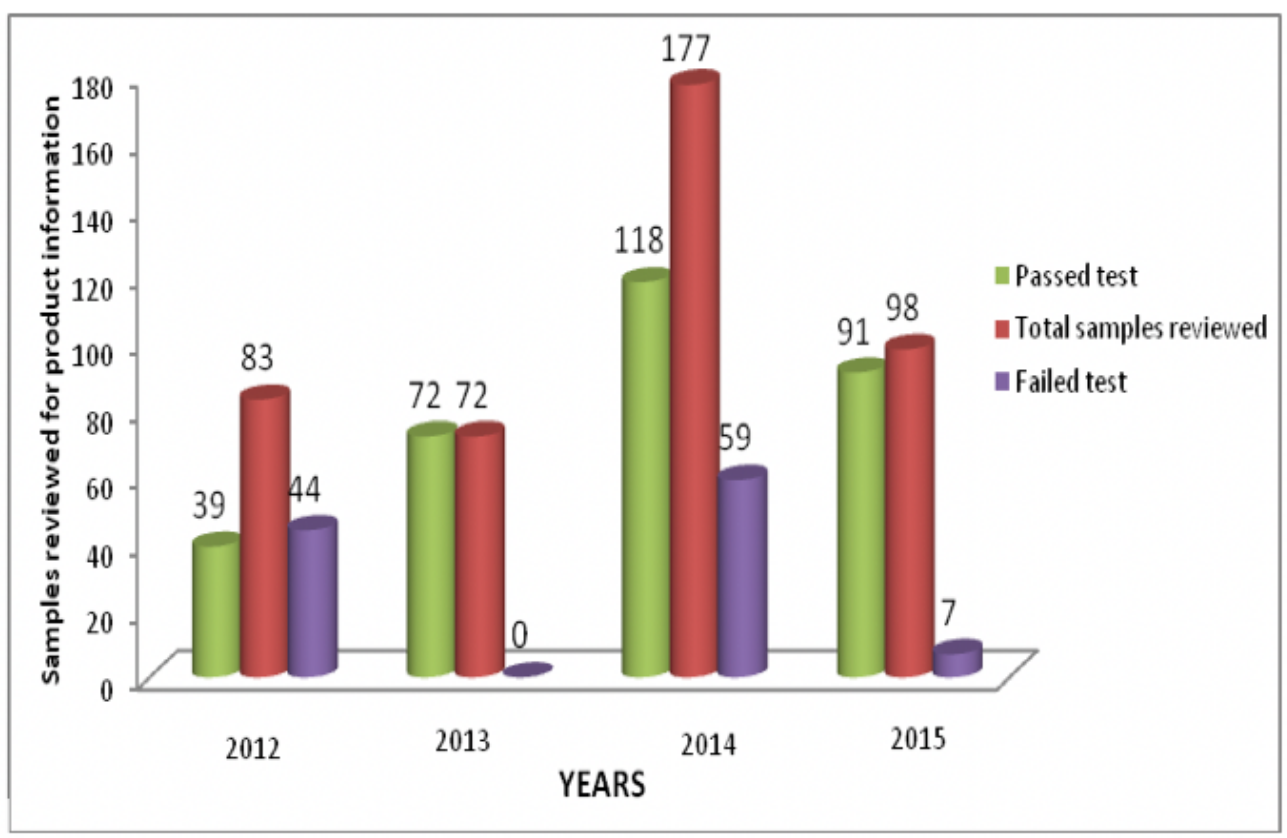

Figure 4

Results of product information review per year

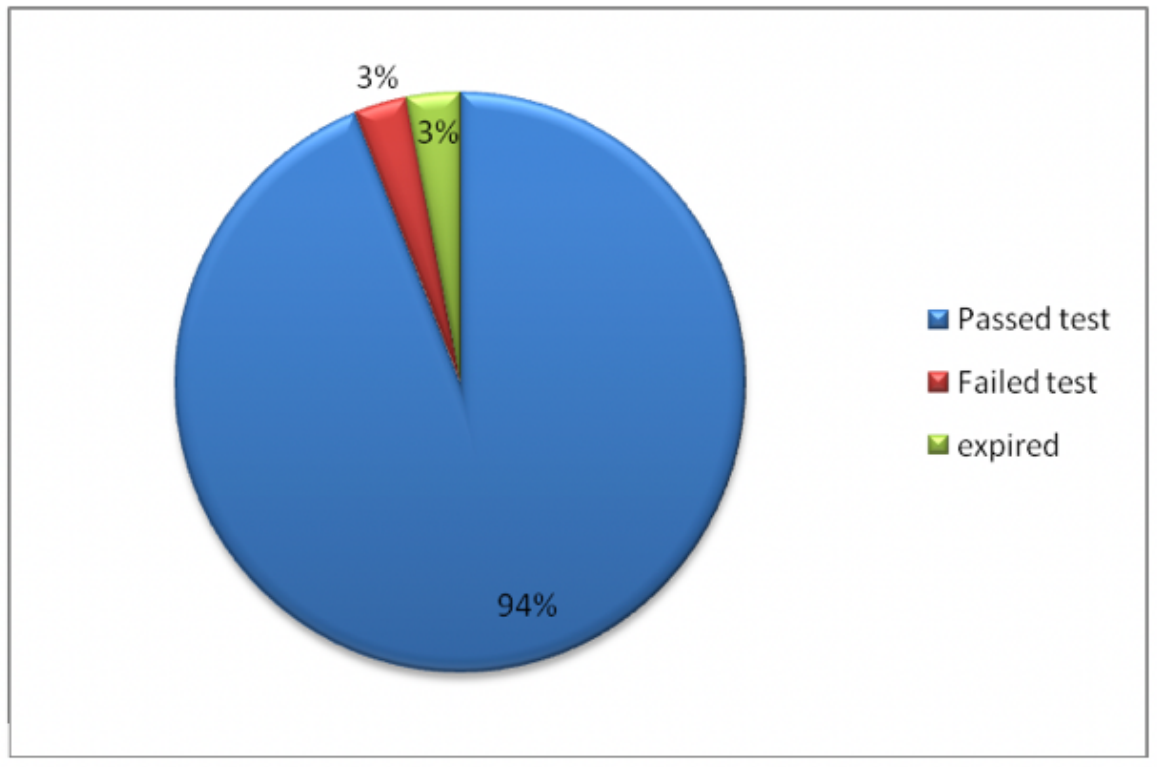

Figure 5

Proportion of samples passed laboratory tier II confirmatory testing

\section{Supplementary Files}

This is a list of supplementary files associated with this preprint. Click to download.

- STROBEchecklistcrosssectionalqualityofARVs.doc 